\title{
COMMENT
}

\section{Miniaturized terahertz-driven electron accelerator}

\author{
Masaki HADA (ه) \\ Tsukuba Research Center for Interdisciplinary Materials Science, University of Tsukuba, Tsukuba, Ibaraki 305-8573, Japan
}

(C) Higher Education Press and Springer-Verlag GmbH Germany, part of Springer Nature 2019

Terahertz-driven (THz-driven) electron acceleration has recently emerged as a promising approach for developing the future compact ultrashort pulsed electron sources. Zhang et al. [1] have developed a segmented terahertz electron accelerator and manipulator (STEAM) and demonstrated the device by employing millimeter-scale drivers in the $\mathrm{THz}$ range. The benefits of high field-gradients and compactness can be realized in a practical acceleration device with a high degree of control and stability. The essential accelerator beam manipulations, including acceleration, compression, streaking, focusing, and deflection, are achieved in a single STEAM device. The STEAM is the first THz-driven device shown to handle moderate charge bunches (up to $20 \mathrm{fC}$ per bunch) and provide significant ( $>50 \%$ ) relative changes in energy with a clean shift of the energy spectrum. These capabilities demonstrate STEAM's usability and represent a significant advancement over previous devices, which either involved few-electron beams or modulated the energy spectrum by a few percents. It also shows its capability as a useful electron source with intrinsic synchronization that provides $100 \mathrm{fs}$ bunches with sufficient charge for ultrafast electron diffraction studies. The small-scaled STEAM device should be critical for future accelerator technology of further pulse-compression and synchronization.

The future of these THz-driven devices is fascinating. Due to the short field-exposure-times and high frequencies of single-cycle $\mathrm{THz}$ pulses, field-gradients of $\sim 1 \mathrm{GV} / \mathrm{m}$ can be expected with sub-mJ $\mathrm{THz}$ pulses that are now available, enabling beam manipulations beyond conventional accelerators.

\section{Reference}

1. Zhang D, Fallahi A, Hemmer M, Wu X, Fakhari M, Hua Y, Cankaya H, Calendron A L, Zapata L E, Matlis N H, Kärtner F X. Segmented terahertz electron accelerator and manipulator (STEAM). Nature Photonics, 2018, 12(6): 336-342

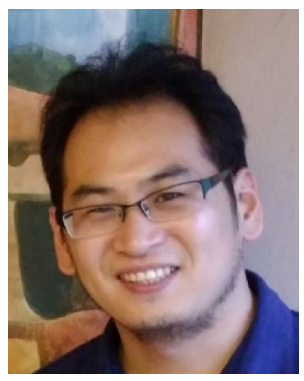

Masaki Hada received the Ph.D. degree from Department of Nuclear Engineering, Kyoto University, Japan in 2010. During 2011-2018, he joined the Universität Hamburg, the Max Planck Institute for the Structure and Dynamics of Matter, Tokyo Institute of Technology, and Okayama University. In 2019, he received the position of associate professor in University of Tsukuba. He has worked on the developments of ultrashort pulsed electron sources and measurements of ultrafast phenomena.

Received December 4, 2019

E-mail: hada.masaki.fm@u.tsukuba.ac.jp 